\title{
A 41-year-old woman with paroxysmal abdominal pain, weight loss and an epigastric bruit
}

\author{
Antonella Marcoccia $\cdot$ Marco Di Tola • \\ Francesca Paris • Giuseppe Donato • \\ Valerio Libanori · Antonio Picarelli
}

Received: 3 June 2009/Accepted: 18 August 2009/Published online: 29 September 2009

(C) SIMI 2009

\section{Case presentation}

Dr. Picarelli: A 41-year-old woman, presenting with a long history of severe recurrent abdominal pain, was admitted to our gastroenterology department.

The pain was intermittent during the prior 4 years occurring at no set time of day or night. The abdominal pain was related neither to food ingestion, bowel movement, nor to flatus or stool evacuation. Exercise and emotional stress frequently caused worsening of the symptoms. In the previous year, she reported at least four episodes of admission to the emergency department (ED) because of severe abdominal pain partially resolving with antispastic therapy.

The patient observed weight loss since the pain motivated a reduced intake of food. As the pain occurred after stress, a psychotherapy was started, but provided no benefit.

Anamnestic data did not show any family history of inflammatory bowel disease, or cancer. The patient had undergone a cesarean section, and as well had an appendectomy when she was age 12 .

Case Report Award, SIMI National Meeting, Genova 2008.

A. Marcoccia ( $\square)$

UOD ANGIOLOGIA, Ospedale Sandro Pertini,

Via Dei Monti Tiburtini, 00157 Rome, Italy

e-mail: antonella.marcoccia@aslromab.it

M. D. Tola · F. Paris · V. Libanori · A. Picarelli Dipartimento di Scienze Cliniche, Policlinico U. I, Università Sapienza di Roma, Rome, Italy

e-mail: antonio.picarelli@uniroma1.it

G. Donato

Dipartimento Medicina Clinica, Policlinico U. I,

Università Sapienza di Roma, Rome, Italy
Abdominal examination disclosed distension and bloating without peritoneal signs, and negative results of inflammatory tests. Occult fecal blood test was negative as well as small bowel $\mathrm{X}$-ray examination.

\section{Differential diagnosis}

Dr. Paris, Dr. Di Tola, Dr. Libanori, Dr. Donato: At the first clinical observation, the patient presented asymptomatic with a soft and non-tender abdomen, without detectable masses. The abdomen auscultation disclosed only a loud systolic bruit in the epigastrium with loss of intensity during deep inspiration.

The patient was tested for lactase deficit by a lactose breath test. A patch test for nickel was negative. Celiac disease was excluded by endomysial and transglutaminase antibodies sera detection. The patient also had a gastroscopy and a colonoscopy that did not show any peptic, inflammatory lesions or tumor.

\section{Preliminary diagnosis}

Dr. Picarelli: Recurrent chronic abdominal pain is frequently present in many gastrointestinal diseases. Nevertheless the patient presented no observable structural or biochemical alterations. The patient was therefore considered to be affected by functional disorders. The diagnosis of irritable bowel syndrome (IBS) or functional dyspepsia was considered, and dietary and pharmacological procedures were undertaken. Proton pump inhibitors, antispastic and antidepressive therapies were administered, but the symptoms did not improve. The patient continued to present abdominal pain with nocturnal weakness. 


\section{Further investigations}

Dr. Marcoccia: Because of the presence of the abdominal bruit, a Duplex sonography abdominal examination was performed. The sonographic examination confirmed the presence of the epigastric bruit and its increasing intensity with deep expiration. The Duplex sonography examination revealed, during deep expiration, a noticeable increase of flow systolic speed in the celiac axis artery $(300 \mathrm{~cm} / \mathrm{s})$. This sonographic alteration, due to the change of emergence angle of celiac axis from aorta, suggested the hemodynamic stenosis of the celiac artery by the arcuate ligament of the diaphragm. A dynamic aortography, subsequently performed, during deep expiration, showed partially inverted flow from the gastroduodenal artery to the hepatic artery trunk. This finding in the celiac artery is an indirect sign of stenosis.

A three-dimensional CT scan confirmed the sonographic findings.

\section{Clinical diagnosis}

Dr. Picarelli, Dr. Marcoccia: The clinical condition known as celiac artery compression syndrome (CACS) can be considered as a cause of recurrent upper abdominal pain when other more common gastrointestinal diagnoses have been ruled out. The upper recurrent abdominal pain, together with the presence of an epigastric bruit, accentuated during deep expiration, highlights the compression of the celiac artery trunk, by the arcuate ligament of the diaphragm. This condition, first labeled as Dunbar syndrome, is now recognized as the CACS. It is characterized by the clinical "triad": paroxysmal abdominal pain, weight loss and an epigastric systolic bruit.

\section{Treatment}

Dr. Marcoccia: The pathogenesis of the pain in CACS is not clearly understood. It seems that dynamic and intermittent compression of the celiac trunk, by the arcuate ligament of the diaphragm, does not explain the pain as an expression of reduced arterial flow, because extensive collateralization via the superior mesenteric artery exists under normal vascular conditions, and especially in young patients. The lack of ischemic signs in the downstream districts, as demonstrated by CT scan, suggests that CACS could be due to a neurovascular cause rather than being caused merely by narrowing of the aortic hiatus and consequential celiac artery compression.

Therefore, the patient, after exclusion of further different causes in the differential diagnosis, because of the severe recurrent abdominal pain, underwent surgical treatment by a laparoscopic procedure. The advantages of a minimally invasive approach are fewer adhesion, decreased intraoperative blood loss, cosmetic benefits and short hospital stay [1-3].

The surgical treatment was performed to the cut the arcuate ligament with the anterior displacement of the celiac artery and the celiac plexus.

\section{Follow-up}

Dr. A. Marcoccia: The period following the surgical treatment was characterized by the complete relief of preoperative symptoms. The duplex ultrasound showed a decreased value and uniform celiac axis speed $(113 \mathrm{~cm} / \mathrm{s}$ during deep expiration) and the angio-CT scan confirmed the successful release of the median arcuate ligament. The patient at follow-up, 17 months later, appeared completely free of symptoms with remarkable enhancement of the quality of her life.

We plan a long-term follow-up with duplex ultrasound and clinical examination every 6 months.

\section{Discussion}

Dr. Marcoccia, Dr. Picarelli: Celiac artery compression syndrome(CACS) is a rare and little known condition characterized by celiac axis compression by the straddling arcuate ligament. This ligament connects both diaphragmatic crura ascending on both sides of the lumbar vertebral column. The first visceral branch of the abdominal aorta is the celiac axis emanating directly beneath this ligamentous arch. In some individuals, the topographic relationships of the neighboring structures are so close that the celiac axis is compressed from above by the ligament. The extent of this compression varies with varying position of the diaphragm, but is typically most pronounced during deep expiration. Sometimes the celiac axis is completely obstructed. There had been a debate on correlation of clinical symptoms with results of imaging procedures. Atherosclerotic narrowing is not a rare finding in asymptomatic individuals with generalized peripheral arteriosclerosis [4].

There has been a long-standing debate about whether restricted celiac perfusion is capable of causing symptoms. Anatomic studies reveal a high incidence of an arteriosclerotic celiac axis narrowing in apparently asymptomatic individuals.

CACS is a diagnosis of a possible cause of recurrent upper abdominal pain, only after other more common diagnoses have been ruled out. It is generally caused by a low insertion of the diaphragmatic crus, or by the 
malposition of the median arcuate ligament. Less common causes of the syndrome are retroperitoneal fibrosis or an aortic aneurysm [5]. Lipshutz [6] was the first to describe the external compression of the celiac trunk in 1917. Harjola [7] hypothesized the connection between compression of the artery and epigastric pain symptoms.

Dunbar [8], presented surgical treatment with decompression of the celiac artery by releasing the median arcuate ligaments compressing the celiac axis during breath expiration, and gave the name to the syndrome as Dunbar's Syndrome.

Persistent symptoms or relapse following surgical treatment for CACS have led investigators to question the etiology of this disease.

Dispute still exists about whether obstructed flow is a sufficient mechanism to explain the symptoms [9]. Despite the fact that the syndrome can cause a clinically variable pain syndrome, the pain experienced by patients cannot, however, be explained by mesenteric ischemia caused by the existing trunk stenosis, because extensive collateralization via the superior mesenteric artery exists under normal vascular conditions especially in young patients. It is furthermore accepted that increased mechanical irritation of the celiac plexus causes a sympathetic autonomic pain response. CACS consists of symptoms caused by a neurovascular narrowing of the aortic hiatus and of the celiac plexus.

Mechanical celiac ganglion irritation was suspected to be mainly involved. [10].

Some authors perform a bisectional nervous tissue removal in addition to excision of some parts of the median arcuate ligament [10].

CACS is a diagnosis of exclusion characterized by the clinical "triad": paroxysmal abdominal pain, weight loss and an epigastric bruit.

Some patients present with non-specific symptoms such as nausea, vomiting, pain independent of time of eating or of the day, weight loss and nocturnal awakening. Frequently patients will present with recurrent diarrhea, distension of superior abdominal quadrants and bloating that can be explained by the irritation of the celiac plexus [11].

When diagnosing CACS, because it is rare, it is prudent to first search for more common diseases such as chronic inflammatory bowel disease, gastric or duodenal ulcers, or cancers.

An epigastric bruit on abdominal auscultation can suggest the presence of the syndrome, and duplex sonography confirms the diagnosis. Color Doppler sonography is the method of choice for exploration of blood visceral vessels when trying to find a CACS. We used this technique to search for characteristic vascular findings at the celiac axis in the same manner as described by Scholbach [10]: asymmetric impingement of the celiac axis by the arcuate ligament with marked variation during respiratory excursions of the diaphragm. B-mode and color Doppler images resembled the angiographic description. Flow velocity measurement as an additional criterion can be obtained. A 7 peak systolic velocity of greater than $200 \mathrm{~cm} / \mathrm{s}$ has a sensitivity of $75 \%$ and a specificity of $89 \%$ in detection of a $70 \%$ or greater celiac axis stenosis $[12,13]$.

In summary, CACS is a rare cause of recurrent epigastric pain (2 per 100,000 patients) [3] that should be included in a differential diagnosis, mainly in younger patients, in whom standard diagnostic procedures are negative. The cause of the paroxysmal pain is often even thought to be psychosomatic. Many of these patients are diagnosed as psychiatric cases with hyperventilation. In such patients it is important to take a full and detailed clinical history, and to perform a careful abdominal examination. After common diseases have been excluded, if an epigastric bruit is present, it is mandatory to perform a duplex sonography with color Doppler during respiratory excursions of the diaphragm.

Here, we have a patient laparoscopically treated with success. The woman remains completely free of symptoms 17 months after the operation and has a notable enhancement in quality of life. The severe epigastric pain ceased immediately after the surgery, and never returned. More experience and a long-term follow-up are desirable to decide when and whether a surgical intervention seems helpful, or whether a conservative non-operative management is effective.

Conflict of interest statement The authors declare that they have no conflict of interest related to the publication of this manuscript.

\section{References}

1. Dordoni L et al (2002) Celiac artery compression syndrome: successful laparoscopic treatment. A case report. Vasc Endovasc Surg 36:317-321

2. Carbonelli AM et al (2005) Laparoscopic management of median arcuate ligament syndrome. Surg Endosc 19:729

3. Thomas F et al (2007) Celiac trunk compression syndrome requiring surgery in 3 adolescent patients. J Pediatr Surg 42:709_ 713

4. Mallek R et al (1993) Duplex Doppler sonography of celiac trunk and superior mesenteric artery: comparison with intra-arterial angiography. J Ultrasound Med 12:337-342

5. Marcoccia A et al (2007) Chronic abdominal pain associated with intermittent compression of the celiac artery. Minerva Gastroenterol Dietol 53:209-213

6. Lipshutz B (1917) A composite study of the celiac axis artery. Ann Surg 65:159

7. Harjola PT (1963) A rare obstruction of celiac artery. Ann Chir Gynaecol Fenn 52:547-550

8. Dunbar JD et al (1965) Compression of celiac trunk and abdominal angina. Am J Roentgenol Radium Ther Nucl Med 95:731-744 
9. Balban DH et al (1997) Median arcuate ligament syndrome: a possible cause of idiopathic gastroparesis. Am J Gatroenterol 92:519-523

10. Scholbach $T$ (2006) Celiac artery compression syndrome in children, adolescents and young adults. Clinical and color Duplex sonographic features in a series of 59 cases. J Ultrasound Med 25:299-305
11. Baldassarre E et al (2007) The laparoscopic approach in the median arcuate ligament syndrome. Swiss Med Wkly 137:353354

12. Nicoloff AD et al (1997) Duplex ultrasonography in evaluation of splanchnic artery stenosis. Surg Clin North Am 77:339-355

13. Moneta GL et al (1993) Mesenteric duplex scanning: a blinded prospective study. J Vasc Surgery 17:79-84 